\title{
Study of Right and Left Asymmetry in Neck Shaft Angle and Neck Length of Femur in Jharkhand Population
}

\author{
Smriti jha ${ }^{1}$, Renu Prasad ${ }^{2}$, A.K Dubey ${ }^{3}$, Narendra Thakur $^{4}$ \\ ${ }^{1}$ Junior resident, Anatomy, Rajendra Institue of Medical Sciences Ranchi,,Jharkhand, India. \\ ${ }^{2}$ Professor and HOD, Anatomy, Rajendra Institue of Medical Sciences, Ranchi, Jharkhand, India. \\ ${ }^{3}$ Associate Professor, Anatomy, Rajendra Institue of Medical Sciences, Ranchi, Jharkhand, India \\ ${ }^{4}$ Professor, Anatomy, Rajendra Institue of Medical Sciences, Ranchi, Jharkhand, India
}

\begin{abstract}
Introduction: The proximal end of femur in human is the object of much attention. Geometry of proximal femur has been identified as risk factor for hip fractures. Anatomical study of femur bone serves helpful data to understand different aspect of clinical disease conditions, including common site of fracture, changes in osteoporosis, associated congenital anomalies as well as medicolegal cases. Extensive studies of normal neck angles have been carried out. The values differ considerably in the reports available.

Materials and Method: In the present study one hundred dry femora from different colleges of Jharkhand (East India) were studied.The parameters that were measured in one hundred dry femora (51 femora from right side and 49 from left side) are: (a) Neck shaft angle and (b) Neck length of femur.

Results : The result of the present study are the mean neck shaft angle of right femora was $131.44 \pm 4.72^{\circ}$ and for left femora was $131.53 \pm 5.29^{\circ}$, the mean neck length of right femora was $29.1 \pm 02.6 \mathrm{~mm}$ and for left femora was $29.3 \pm 03.1 \mathrm{~mm}$.

Conclusion: All the clinician must be familiar with normal neck- shaft angle for better comprehension of clinical and pathological states of hip joint .The neck- shaft angle ,neck length and other parameters are important to design prosthesis for hip replacement.In the present study the mean neck shaft angle of the left femora was feebly higher than in the right femora but statistically not significant ( $p>0.05)$. The mean femoral neck length of left femora was feebly higher than the right femora, but statistically nonsignificant ( $p>0.05)$.
\end{abstract}

Keywords: Neck-shaft angle,Neck length and femur, Prosthesis, Goniometer.

\section{Introduction}

The proximal end of femur in human is the object of much attention. The knowledge of its anatomy is a prerequisite for complete understanding of the mechanics of the hip joint and serves as a basis for the treatment of pathological conditions of the hip and femur. ${ }^{1}$ Extensive studies of normal neck angles have been carried out. The hip joint is one of the largest and most stable joints in the body. The joint depends on the angle formed between the neck of the femur and diaphysis that keep the inferior limb more distant from the pelvis and facilitates movement at the hip joint. ${ }^{2}$ The femur is the lonest and strongest bone of thigh in human . It is about $45 \mathrm{~cm}$ long in an average individual. Like any other long bone it has two ends upper and lower, and a shaft. The upper end bears a rounded head whereas the lower end is widely expanded to form two large condyles. The head is directed medially upward from its short neck. The femoral neck length is approximately 5 $\mathrm{cm}$ and connects the head to the shaft at an average angle of $135^{\circ}$. The neck shaft angle has been related to mechanical strength of proximal femur. It is also named as Angle of inclination, Collodiaphyseal angle and Cervicodiaphyseal angle. ${ }^{3}$ The neck shaft angle of femur is the obtuse angle formed by intersection of the femoral shaft axis with the femoral neck axis. ${ }^{4}$ The neck shaft angle varies with age ,stature and width of pelvis. When this angle $>135^{\circ}$, condition is known as coxavalga. When angle is $<120^{\circ}$, it is known as coxa vara. The angle of femoral neck is reduced with age .In early infancy the neck shaft angle is about $150^{\circ}$, in childhood $140^{\circ}$, in adult about $125^{\circ}$ and in elderly about $120^{\circ} .{ }^{5}$ The clinical importance of neck shaft angle of femur lies in the diagnosis, treatment and follow up of fractures of the neck of femur, trochanteric fractures, slipped upper femoral epiphysis, development dysplasia of the hip and neuromuscular disorders of the lower extremity. The knowledge of normal asymmetry of right and left neck shaft angle of femur may be of great value in evaluation of patient with known or assumed pathological conditions and in correctional osteotomies in case of femoral fractures. The neck shaft angle can be estimated from a proximal femoral fragment and the required size of the length of the neck can be determined to design the prostheses for the restoration of normal neck shaft angle. ${ }^{6}$ 


\section{Materials And Methods}

Materials for the study consisted of one hundred dry femora, Sliding calliper, Goniometer, scale and pencil. The dry femora were collected from different medical colleges of Jharkhand (East India). Specimen taken under the study were devoid of any Osseous pathology, Previous fracture, Burnt and Abnormal bones and Bones of children .

The Neck-shaft angle is defined as the angle formed between neck axis and long axis of the shaft of femur. ${ }^{1}$ (Photograph 1)

- The neck axis was drawn through the midpoint of the neck.

- The shaft axis was drawn through the midpoint of narrowest part of the shaft.

- The angle formed between these two axes measured by goniometer.

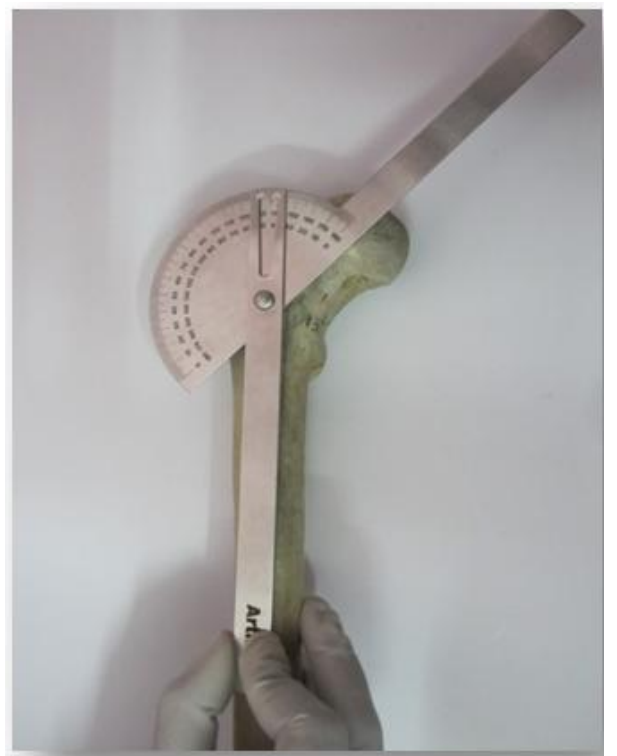

Photograph 1: showing measurement of neck shaft angle of femur

The Neck length was measured from anterior aspect. The line joining the midpoint taken at the junction of the head and neck and midpoint of the intertrochanteric line was measured with the help of sliding calliper.(Photograph 2)

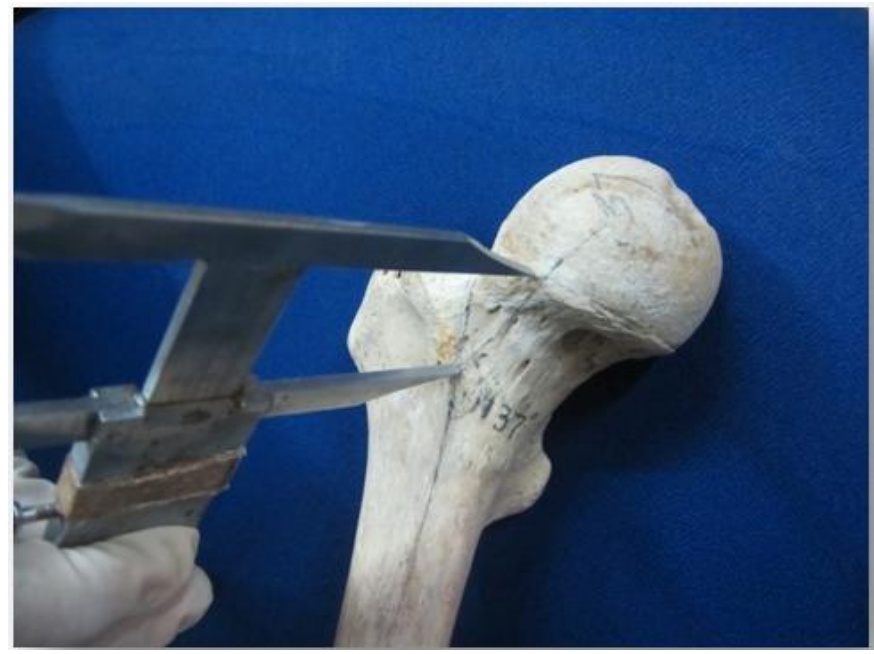

Photograph 2: showing measurement of neck length of femur

\section{Results}

The mean neck-shaft angle and neck length of right and left femora were compared irrespective of sex. In femora the mean neck shaft angle was $131.48 \pm 5.005^{\circ}$, in the right femora $131.44 \pm 4.72^{\circ}$ and in the left femora $131.53 \pm 5.29^{\circ}$. In the femora the mean neck length was $29.2 \pm 3.3 \mathrm{~mm}$, in the right femora $29.1 \pm 2.6 \mathrm{~mm}$ and in the left femora $29.4 \pm 3.1 \mathrm{~mm}$. 
Table 1: Showing mean values of femoral neck shaft angle and neck length

\begin{tabular}{|l|l|l|l|}
\hline Parameter & Number & Mean \pm SD \\
\hline \multirow{3}{*}{ Neck shaft angle } & Total & 100 & $131.48 \pm 5.005^{\circ}$ \\
\cline { 2 - 4 } & Right & 51 & $131.44 \pm 4.72^{\circ}$ \\
\cline { 2 - 4 } & Left & 49 & $131.53 \pm 5.29^{\circ}$ \\
\hline \multirow{3}{*}{ Neck length } & Total & 100 & $29.2 \pm 3.3 \mathrm{~mm}$ \\
\cline { 2 - 4 } & Right & 51 & $29.1 \pm 2.6 \mathrm{~mm}$ \\
\cline { 2 - 4 } & Left & 49 & $29.4 \pm 3.1 \mathrm{~mm}$ \\
\hline
\end{tabular}

\section{Discussion}

In the present study the mean neck shaft angle in the femora was $131.48 \pm 5.005^{\circ}$, in the right femora $131.44 \pm 4.72^{\circ}$ and in the left femora $131.53 \pm 5.29^{\circ}$. The mean neck-shaft angle of the left femora was feebly higher than the right side, which was satistically nonsignificant $(\mathrm{p}>0.05)$. This was very similar to the earlier South Indian study by Singh(1986) who found mean neck shaft angle for the left femora $131.3 \pm 3.9^{\circ}$ and for the right femora $131.0 \pm 3.6^{\circ} .^{7}$ Similarly Subhash gujar(2013) also found $136.6 \pm 5.45^{\circ}$ for left femora and $136 \pm 6.68^{\circ}$ for the right femora.$^{8}$ In another study by Issac (1997) in South Indian population the mean neck shaft angle found on the left side $126.5^{\circ}$ and on the right side $126.9^{\circ} .6$ The Shakil Mohammad (2014) found mean neck shaft angle of right femora was $137.44^{\circ}$ and of left femora was $136.9^{\circ} .{ }^{9}$

The mean neck length in the present study was found $29.2 \pm 5.2 \mathrm{~mm}$, in the right femora $29.1 \pm 02.6$ and in the left femora $29.3 \pm 03.1 \mathrm{~mm}$. The values obtained was closer to the Issac (2003) study in South Indian population, $28.6 \mathrm{~mm}$ for right side and $28.1 \mathrm{~mm}$ for the left side. Da Silva (2003) found mean neck length for the right femora $22.3 \pm 3.3 \mathrm{~mm}$ and for the left femora $23.5 \pm 3.6 \mathrm{~mm}$ in the Brazilian population. ${ }^{2}$ Subhsh Gujar (2013) found mean neck length for the right femora $34.5 \pm 4.0 \mathrm{~mm}$ and for the left femora $34.2 \pm 3.62 \mathrm{~mm}$ in Gujarat (India). Shakil Mohmad Khan and Shaik Hussain (2014) found mean neck length $36.1 \pm 4.1 \mathrm{~mm}$ for the right femora and $36.4 \pm 4.3 \mathrm{~mm}$ for the left femora in South Indian population. The mean neck length of the present study $(30.7 \mathrm{~mm})$ was closer to the mean neck length found by D.Ravichandran $(31.8 \mathrm{~mm}) .^{10}$

\section{Conclusion}

The neck- shaft angle of femur allows greater mobility of the femur at the hip joint .All the clinician must be familiar with normal neck- shaft angle for better comprehension of clinical and pathological states of hip joint. The neck- shaft angle, neck length and other parameters are important to design prosthesis for hip replacement. Considering the above mentioned importance the present study was conducted to assess the neck length and neck- shaft angle of femur, its variation with respect to side.

In the present study the mean neck shaft angle in the femora was $131.48 \pm 5.005^{\circ}$, in the right femora $131.44 \pm 4.72^{\circ}$ and in the left femora $131.53 \pm 5.29^{\circ}$. The mean neck shaft angle of the left femora was feebly higher than in the right femora but statistically not significant $(\mathrm{p}>0.05)$. So there is no significant difference between mean neck shaft angle of right and left femora.

The mean neck length in the present study was found $29.2 \pm 5.2 \mathrm{~mm}$, in right femora $29.1 \pm 2.6 \mathrm{~mm}$ and in the left femora $29.3 \pm 3.1 \mathrm{~mm}$.. There was no significant side difference in the neck length. No positive correlation was found between mean neck length and mean neck shaft angle.

This study will be helpful in designing prosthesis for hip replacement, in the diagnosis of various hip pathologies and in the determination of racial variations.

\section{References}

[1]. Olav Reikeras, Arne Holseth, et al.Femoral Neck Angles. Acta Orthopaedica Scandinavica 1982;53:775-779

[2]. Da Silva VJ.Oda JY and Sant'ana DMG . Anatomical aspects of proximal femur of adults Brazilians.Int .J .Morphol 2003;21(4) 303-8.

[3]. John Y Anderson and Erik Trinkaus. Pattern of Sexual bilateral and interpoulational variations in human femoral neck shaft angles.Vol.192. 1997 .p.279-285.

[4]. Delaunay S ,Dusault RG, et al. Radiographic measurements of dysplastic adult hip 1997 .p.78-80.

[5]. Susan Standring. Femur. Grays Anatomy. The Anatomical basis of clinical practice 40 ${ }^{\text {th }}$ Edn 2008.p.1360-65

[6]. Issac, Vettivel, Prasad, Jeyaseelan, and Chandi,G. Prediction of the femoral neck-shaft angle from the length of the femoral neck.Clin.Anat.,1997; 10:318-323.

[7]. Singh P.I, Bhasin M.L.Anthropometry $1^{\text {st }}$ Edn;Delhi Educational Publishers and Booksellers 1968 pp 142.

[8]. Gujar Subhash, Sanjay Vikani, Jigna Parmar, K VBondre. A correlation between femoral neck shaftangle to femoral neck length. IJBAR;2013:04(05).

[9]. Shakil Mohamad Khan and Shaik Hussain Saheb . Study on neck-shaft angle and femoral length of South Indian femurs.Int J Anat Res2014, Vol2(4):63-35

[10]. D.Ravichandran ,N. Muthukumaravel, R.Jaikumar et al. Proximal femoral geometry in Indians and its clinical applications.J.Anat.Soc.India60(1)6-12(2011) 